\title{
Sequencing of RAS/RAF pathway genes in primary colorectal cancer and matched liver and lung metastases
}

\author{
Nikki Knijn ${ }^{1}$, Carlijn van de Water $^{1}$, Shannon van Vliet ${ }^{1}$, Jos Meijer ${ }^{2}$, Sietske Riemersma ${ }^{3}$, Maria Tebar ${ }^{3}$, \\ Cornelis Punt ${ }^{4}$, Leonie Mekenkamp ${ }^{5}$, Femke Simmer ${ }^{1}$ and Iris Nagtegaal ${ }^{1 *}$
}

\begin{abstract}
Background: Mutations in the RAS/RAF pathway predict resistance to anti-epidermal growth factor receptor antibodies in colorectal cancer (CRC), and may be targets for future therapies. This study investigates concordance of BRAF, HRAS, KRAS, NRAS and PIK3CA mutation status in primary CRC with matched liver $(n=274)$, lung $(n=114)$ or combined liver and lung metastases $(n=14)$.

Methods: Next generation sequencing was performed on DNA from formalin-fixed paraffin embedded CRC and matched liver and/or lung metastases, for recurrent mutations in BRAF, HRAS, KRAS, NRAS and PIK3CA and using the single-molecule molecular inversion probe method.

Results: Paired sequencing results on all five genes were reached in 249 of the 402 cases (62\%). The obtained number of unique reads was not always sufficient to confidently call the absence or presence of mutations for all regions of interest. The mutational status of matched pairs was highly concordant; $91.1 \%$ concordance for all five genes, 95.5\% for KRAS, 99.1\% for NRAS. Lung metastases more often harboured RAS mutations compared to liver metastases (71\% vs. $48 \%, p<0.001)$.

Conclusions: In this large series of CRC we show that both primary tumors and corresponding metastases can be used to determine the mutational status for targeted therapy, given the high concordance rates. Next generation sequencing including a single molecule tags is feasible, however in combination with archival formalin-fixed paraffin embedded material is limited by coverage depth.
\end{abstract}

Keywords: Colorectal cancer, Liver metastases, Lung metastases, Next generation sequencing

\section{Background}

Monoclonal antibodies against the Epidermal Growth Factor Receptor (EGFR) are nowadays firmly established within treatment regimens for patients with metastatic colorectal cancer $(\mathrm{CRC})$. These antibodies inhibit ligand induced stimulation of several intracellular signalling pathways such as RAS/RAF/MAPK and phosphoinositide-3 (PI3K) pathway, which results in decreased stimulation of cell cycle progression, proliferation, angiogenesis and stimulation of apoptosis. The presence of activating

\footnotetext{
* Correspondence: Iris.Nagtegaal@radboudumc.nl

${ }^{1}$ Department of Pathology, Radboud Institute of Molecular Life Sciences, Radboud University Medical Center, PO Box 9101, Nijmegen 6500 HB, The Netherlands

Full list of author information is available at the end of the article
}

mutations in the RAS/RAF signalling pathway limits the effects of this treatment [1-3]. It is therefore standard procedure to perform molecular testing in order to determine the indication for these types of therapy. In previous work we have shown that for a limited number of KRAS mutations there is minimal discordance between primary CRC and liver metastases [4]. However, we performed conventional Sanger sequencing and did not test all relevant $R A S$ genes, nor BRAF and PIK3CA genes.

Next generation sequencing is increasingly performed in daily clinical practice. One advantage over Sanger sequencing is that low-frequency mutations can be detected. However, application of enrichment methods to gain sufficient quantities of DNA may result in amplification bias. To overcome this issue single molecule tags

(C) The Author(s). 2019 Open Access This article is distributed under the terms of the Creative Commons Attribution 4.0 International License (http://creativecommons.org/licenses/by/4.0/), which permits unrestricted use, distribution, and 
can be used [5, 6]. Consequently, in the current study we applied a single-molecule molecular inversion probe (smMIP)-based next generation sequencing approach to investigate concordance rates for all relevant $B R A F$, HRAS, KRAS, NRAS and PIK3CA mutations in CRC with matched lung and liver metastases.

\section{Methods}

\section{Patient selection}

All patients with histologically confirmed CRC who underwent surgical resection of the primary tumor and a sufficient biopsy or surgical resection of the corresponding liver or lung metastasis between 1984 and 2011 were included in this analysis. In case of multiple primary tumors or metastases, all material was used for sequencing. Formalinfixed-parafin-embedded (FFPE) material from three large pathology laboratories; Radboud university medical center, Nijmegen, Rijnstate hospital, Arnhem and Laboratory of Pathology East Netherlands, Hengelo, was used. The Institutional Review Board of the Radboud university medical center, Nijmegen, ruled that the current study does not require informed consent according to Dutch law, but based on national guidelines for the use of archival material, the Institutional Review Board agrees with the study proposal (CMO 2013/048, date 23/4/2013).

\section{DNA extraction and mismatch repair status analysis}

FFPE tissue blocks were cut at $4 \mu \mathrm{m}$ thickness and stained with haematoxylin and eosin (HE). The slide with highest tumor cell percentage was selected and the presence of tumor was marked by an expert pathologist (IN). Samples with tumor cell percentages above $30 \%$ were included. Subsequently the blocks were cut at $20-40 \mathrm{~mm}$ thickness and macrodissected for DNA extraction. DNA was extracted according to the manufacturer's protocol (QIAamp DNA Micro Kit, Qiagen, Hilden, Germany), and DNA concentration was determined with Qubit (2.0, Life Technologies, Foster city, CA, USA). For each sample approximately 250 nanogram of DNA was included.

Mismatch repair status analysis was assessed by immunohistochemistry. Microsatellite instability (MSI) analysis was performed in all cases with absence or unequivocal protein expression, using five microsatellite markers (NR21, NR24, NR27, BAT 25 and BAT 26, pentaplex PCR system). A tumor was defined as MSI if at least two of the five markers showed instability [7].

\section{Mutational analysis smMIP sequencing}

smMIP based sequencing was used to detect mutations in BRAF (exon 15, targeted codons D594-K601), HRAS (exon 2, targeted codons G12, G13, exon 3, targeted codons, A59, Q61), KRAS (exon 2, targeted codons G12, G13, exon 3, targeted codons A59, Q61, exon 4 targeted codons K117, A146), NRAS (exon 2, targeted codons
G12, G13, exon 3, targeted codons A59, Q61, exon 4 targeted codons K117, A146), and PIK3CA (exon 10, targeted codons E542-Q546, exon 21, targeted codons M1043-G1049). This technique is clinically validated and implemented in the routine diagnostics workflow of our hospital and is extensively described elsewhere [6]. In short, smMIPs are long oligonucleotides consisting of two targeting arms, the extension probe and the ligation probe, joined by a backbone including a single molecule tag. The probe sequences are complementary to the sequences surrounding the target region. During the capture reaction, the smMIP mixture is hybridized to the DNA, followed by gapfilling through extension and ligation, resulting in a circular smMIP. Exonuclease treatment removes all linear DNA. The circular smMIP are amplified by PCR using barcoded primers recognizing sequences in the backbone of the smMIP. After target enrichment, products were pooled with comparable amounts of the smMIP enriched products (based on an agarose gel) and sequenced on a NextSeq500 instrument (Illumina, San Diego, CA, USA). The commercial analysis software Sequence Pilot (JSI medical systems, Ettenheim, Germany) was used for variant identification. Sequencing reads are aligned and reads sharing the same unique single molecule tag are merged into a consensus read sequence. This reduces the number of sequencing artifacts, mutations present in the genomic template are maintained. The settings as described by Eijkelenboom et al. [6] were used for generating the consensus reads and for variant calling. The transcripts for variant annotation were: BRAF ENST00000288602; KRAS ENST00000311936; PIK3CA ENST00000263967; HRAS ENST00000451590; NRAS ENST00000369535. After variant calling using the commercial software, all variants were manually inspected and curated based on Cosmic (Cosmic v.81 (May 2017), Sanger Institute) and Alamut (AlamutVisual 2.9.0 (Dec. 2016), Interactive Biosoftware). Furthermore, in the downstream analysis the minimum mutant allele frequency was set at $5 \%$. In fact, $96 \%$ of the variants selected had an allele frequency greater than or equal to $10 \%$. Therefore, the minimum absolute coverage to reliably exclude the presence of mutations was set at 125 combined. This threshold excludes, with an approximate certainty of $>90 \%$, the presence of a mutation at minimally $10 \%$ mutant allele frequency within the covered regions.

\section{Statistical analysis}

To compare patient and primary tumor characteristics between patients with wild-type and mutant sequencing results Wilcoxon's rank sum test or $\chi^{2}$ for numerical or categorical variables, respectively was used. Differences in mutation status between the primary tumor and corresponding metastasis were analyzed by calculating the 
percentage of concordance and discordance. Concordance was defined as both primary tumor and metastasis having no mutations (wildtype-wildtype) or exactly the same mutation (mutation-mutation, same variant). Discordance was defined as a mutation in either tumor or metastasis which was not found in the corresponding counterpart (wildtype-mutant, mutant-wildtype) or as two different mutations in tumor and metastasis (mutant-mutant, different variants). Statistical analyzes were performed using the statistical software package SPSS 20.0 (SPSS Inc., Chicago, Illinois, USA). Differences were considered to be statistically significant with a $P$-value below 0.05 . All statistical tests were two-sided.

\section{Results}

A total of 402 patients with CRC were included, 274 with liver metastases, 114 with lung metastases and 14 with both liver and lung metastases. The majority of patients presented with a solitary liver metastasis (253 patients), 10 patients had multiple liver metastases (range 2-5), nine patients had two primary tumors together with one liver metastasis and two patients had two primaries and two liver metastases sequenced. In most patients with lung metastases $(n=103)$ one metastasis was available for testing, nine patients had two lung metastases and two patients presented with two primaries and one lung metastasis.

\section{Concordant cases}

"In the paired samples with high enough coverage for all five genes, the overall concordance rate for these five genes together was $91.1 \%$ (216 concordant/237 samples in total)." The individual concordance rate for $B R A F$ was 99.6\% (246/247), for HRAS 100\% (364/364), for KRAS 95.5\% (193/202), for NRAS 99.1\% (228/230) and for PIK3CA 94.7\% (213/255). All discordant cases for BRAF and NRAS were based on mutations present in the primary tumor, as were the majority of the KRAS (6/7) and PIK3CA discordant cases (9/13) (Fig. 1). The observed discordance in $R A S$ was clinically relevant (i.e. would result in a different treatment approach) in 9 patients (3.9\%).

\section{Differences between liver and lung metastases}

The overall mutation frequency in patients with lung metastases was higher than the mutation frequency in patients with liver metastases (Fig. 2).

The concordance rate in mutational status for all five genes was not statistically different for tumors with liver metastases and tumors with lung metastases (91.2\% (166 concordant/182 total) vs. $89.1 \%$ (49 concordant/55 total), $p=0.64$ ). The rate of MSI was relatively low (2.2\%), all patients with lung metastases were MSS and only eight patients with liver metastases showed MSI (3.2\%).

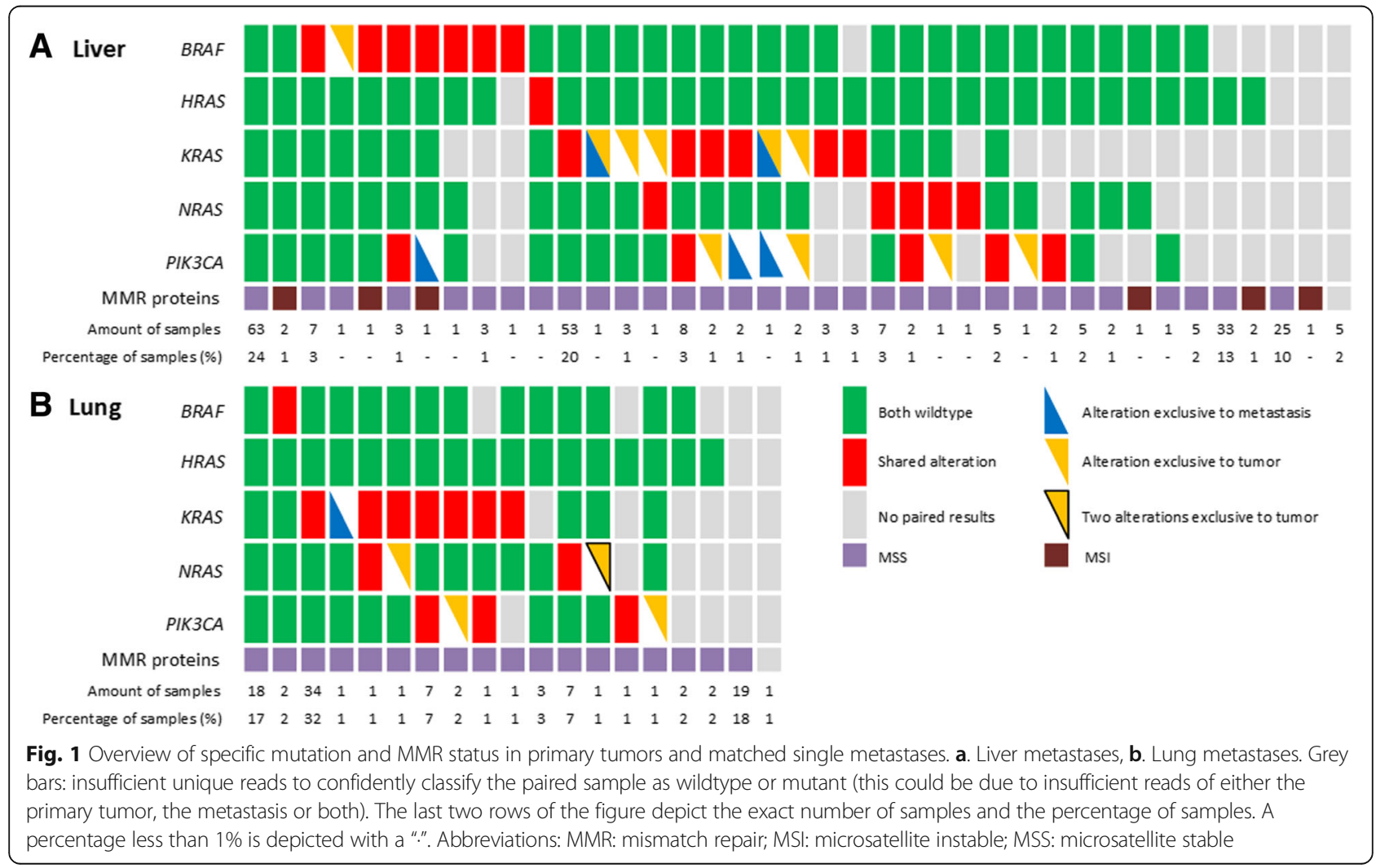




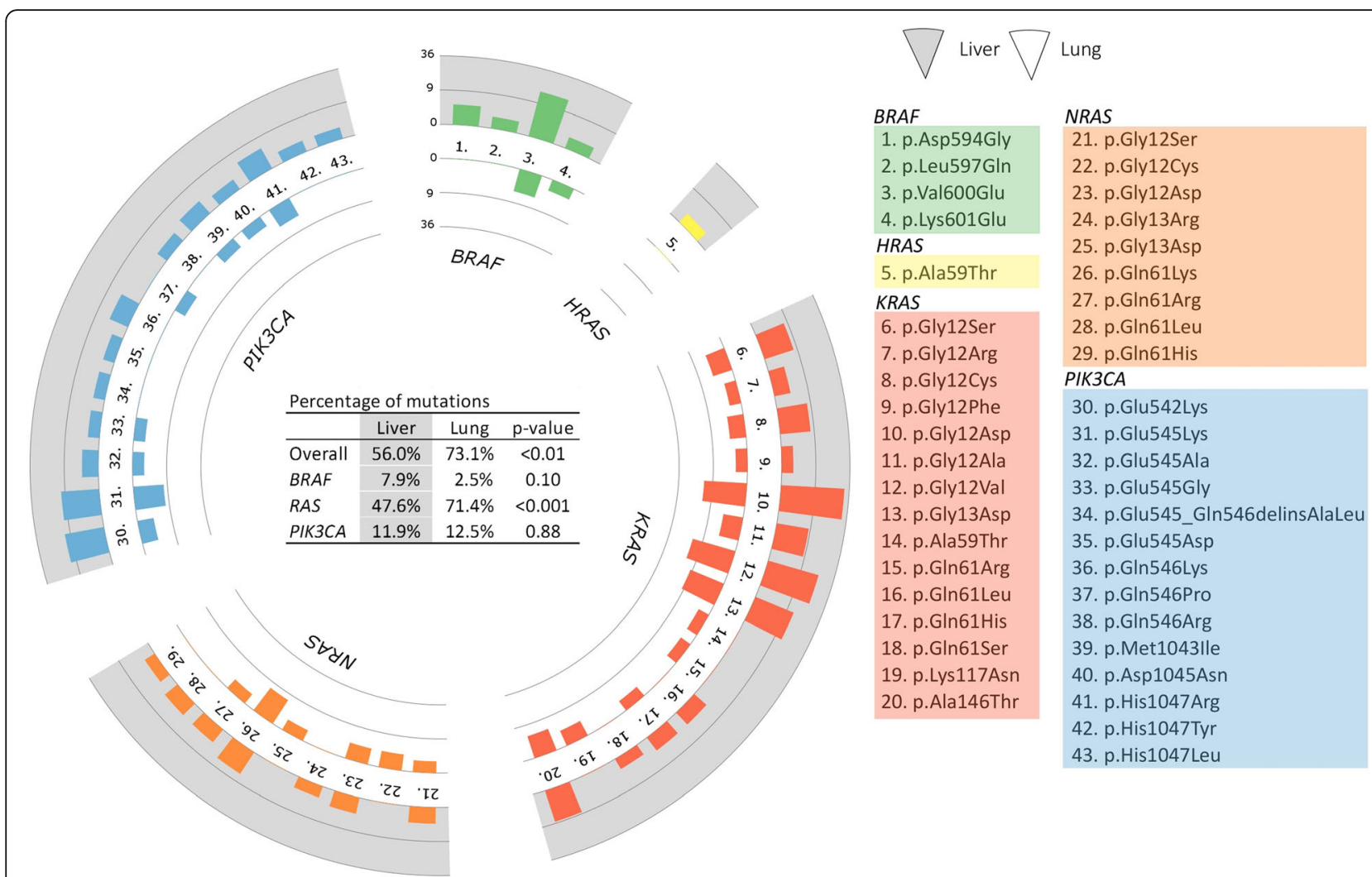

Fig. 2 Overview of specific mutations found in patients with liver and lung metastases. An overall mutation frequency in percentages is given for different genes in the table. For specific mutations, the actual number of mutations is depicted. *Patients with both liver and lung metastases are excluded

\section{Multiple metastases}

In 10 of the 14 patients with liver and lung metastases we obtained sequencing results of all tumor samples. In four patients we did not retrieve sufficient unique sequence reads (in three due to the primary tumor, in one due to failure of all samples). Identical results in mutation status of primary tumor, liver and lung metastases were observed in 9 of the 10 patients (Fig. 3a).

In 10 of the 21 patients with multiple liver or lung metastases, sequencing results were obtained for both primary tumor and metastases. Insufficient sequence coverage was due to failure of all samples (two patients), failure of primary tumor (six patients) or failure of metastases (three patients). Seven of the ten patients showed concordance of primary tumor and metastases (Fig. 3b,c). Discordance was due to mutations limited to the primary tumor (one case) and to the metastases (two cases).

\section{Coverage}

With our settings, we obtained paired sequencing results in $62.0 \%$ (249 concordant/402 total). The minimum coverage depth was reached for BRAF, HRAS, KRAS, NRAS and PIK3CA in 68,72, 58, 58 and $63 \%$ of the 415 primary tumors and in $80,83,68,70$ and $74 \%$ of the 443 metastases (Fig. 4). According to the type of material analyzed, metastases are more often complete on all five genes $(69 \%$ of metastases vs. $59 \%$ of primary tumors, $p=0.003)$.

\section{Comparison with sanger sequencing}

In all patients $K R A S$ Sanger sequencing was performed for exon 2. Good quality results of both Sanger and smMIP sequencing were available for 292 patients. In 81 patients results were only obtained with Sanger sequencing; coverage for the smMIP analysis was too low. In 29 patients results were only obtained with smMIP sequencing. When results from both techniques were available $(n=292)$, concordance was $100 \%$.

\section{Discussion}

This is the first large study that compares mutation status between primary tumors and their corresponding liver and lung metastases using a single molecule tag approach. We observed an overall concordance rate of $91 \%$ in all five genes in paired samples. Concordance rates above $99 \%$ were reached for $B R A F, H R A S$ and NRAS, concordance for KRAS and PIK3CA were 96 and 

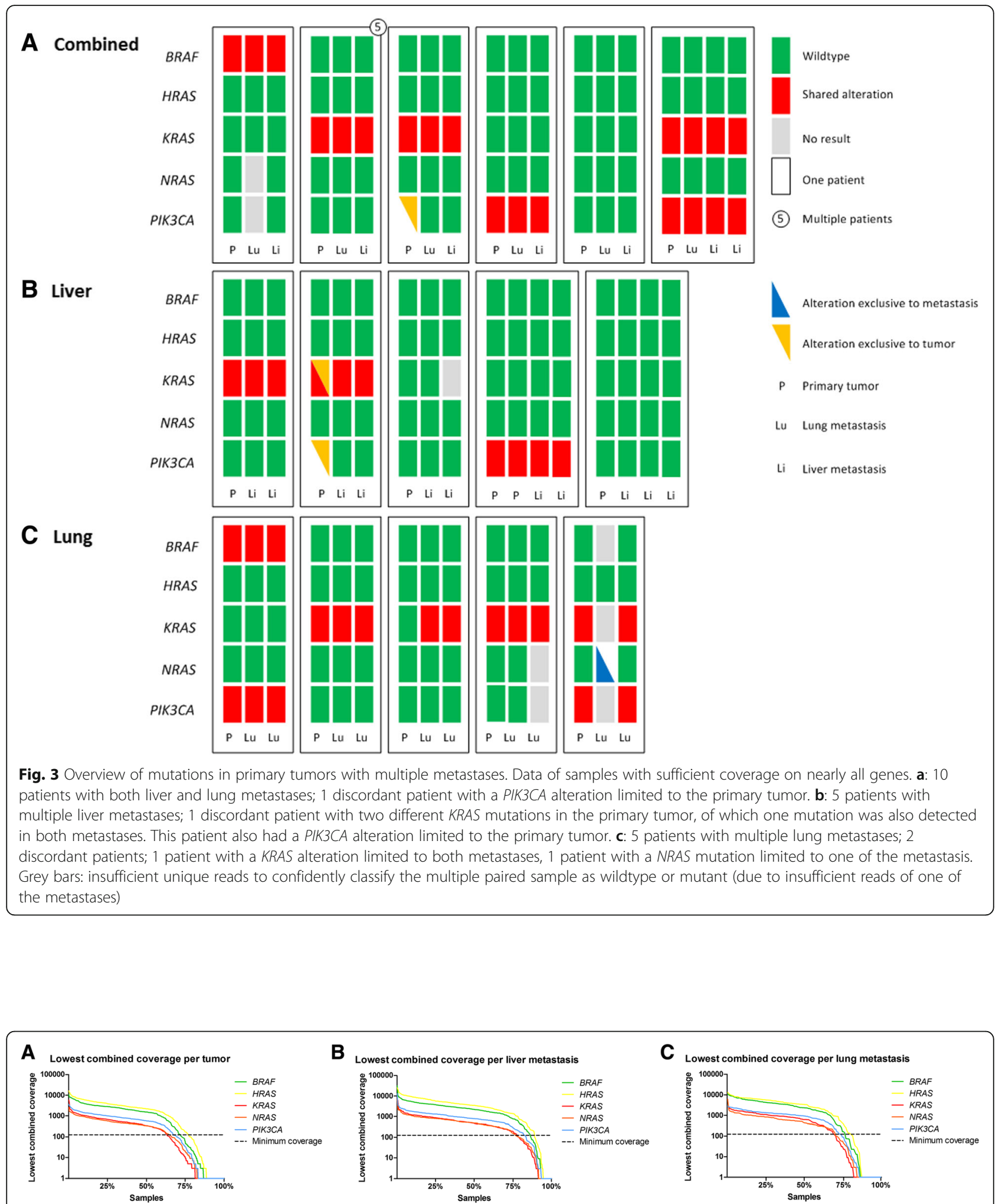

Fig. 4 Lowest coverage per tissue type. a: primary tumor, b: liver metastasis, c: lung metastasis. Y-axis: the lowest coverage of samples in unique reads. Dotted line: the minimum absolute coverage of 125 unique reads $x$-axis: the percentage of samples. In all samples HRAS mutation analysis performs better (coverage above 125 unique reads in the highest percentage of samples), followed by BRAF, PIK3CA, and NRAS/KRAS. Sequencing of liver metastases more often result in enough coverage ( $75 \%$ of samples), followed by lung metastases (70\% of samples) and primary tumors ( $60 \%$ of samples) 
95\%, respectively. Comparable concordance rates for $K R A S$ and BRAF are described in three smaller series [8-10]. In contrast, a discordance rate of $23 \%$ for KRAS and $7 \%$ for $B R A F$ was detected in a study of 43 primary tumors and matched liver metastases [11]. Although an increased KRAS discordance rate was previously reported in CRC with lung metastases [12], we did not observe a difference in concordance rate between liver and lung metastases. These high concordance rates implicate that, in the treatment-naïve setting, there is no need for additional biopsies from metastatic sites in order to obtain a molecular profile to decide on antiEGFR therapy. This is an important message, given the impact of additional interventions on patients, like shown in the meta-analysis of CT guided lung biopsies with overall complication rates of 24 to $38 \%$ [13]. Next to the increased costs and complications, the delay due to additional biopsies and subsequent testing might be considerable. Mutation analysis based on the primary tumor, would have incorrectly withhold anti-EGFR treatment to 8 patients $(3.4 \%)$ and one patient $(0.5 \%)$ would have incorrectly received anti-EGFR therapy. Nevertheless, acquired resistance after anti-EGFR therapy, with novel KRAS or NRAS mutations in 38 to $60 \%$ of cases indicate the necessity of additional biopsies in that setting [14-16]. We observed mutations in the RAS/RAF pathway in $62 \%$ of the metastases, ranging from $73 \%$ in lung metastases and $56 \%$ in liver metastases. The difference between mutation frequency in liver and lung metastases is mainly caused by the higher incidence of RAS mutations in lung metastases (71\% vs. $47 \%)$. This high occurrence of RAS mutations in colorectal cancer with lung metastases is in line with literature $[12,17,18]$. KRAS mutations are also increased in colorectal bone and brain metastases [19]. Distinct metastatic patterns are observed for $B R A F$ mutations as well, which are mainly associated with peritoneal and distant lymph node metastases [20, 21]. Furthermore, as expected [22, 23], a low percentage of MSI was observed, and only in patients with liver metastases (3.2\%). This confirms the inverse relation between MSI CRC and lung metastases [21]. MSI/BRAF mutated CRC seems to have a preference for widespread disease including peritoneal metastases, thus falling outside our current selection and explaining our relatively low incidence of MSI.

Our study illustrates the main limitation of next generation sequencing using single molecule tags, which is coverage dependency. This is even more apparent in our study because we need good coverage for all five genes in two or more samples per patient. In clinical setting limited coverage for an exon of a gene that is not directly involved in resistance to therapy, is less often a problem. However, this is potentially important since a test is only as strong as its lowest coverage. Sufficient unique sequence reads are required to reliably identify the presence or absence of a mutation. Adjusting the settings would increase the number of false positive and false negative calls. We have chosen a certainty level of $>90 \%$ to reliably identify or exclude the presence of a mutation. Currently, there are no guidelines on what level of certainty is acceptable in the molecular setting. Since this has potentially major impact on important treatment decisions, it is vital that oncologists become aware of this issue.

In our study the insufficient coverage was most likely a result of fragmented DNA due to FFPE fixation. Additional sequencing of the same cases did not result in a large improvement of coverage, probably because of this fragmented DNA. All archived material was obtained from a wide timeframe, ranging from 1984 to 2011 . Although subanalysis did not show any correlation between year of resection and completeness of sequencing, older samples are believed to have more fragmented DNA [24, 25].

\section{Conclusion}

In colorectal cancer it is a standard procedure to perform molecular testing to determine $R A S / R A F$ mutation status for targeted therapy. However, whether to test primary tumor or metastasis remains a matter of debate. In our manuscript, we have performed next generation sequencing including single molecule tags of the RAS/ RAF pathway related genes in a large set of primary colorectal cancer cases with matched liver and lung metastases $(n=402)$. With this unique technique, we observed a high concordance in $R A S / R A F$ mutation status between tumors and corresponding metastases. Therefore, discordance in mutation status of anti-EGFR related genes is not an issue for molecular testing in treatment-naive CRC.

\section{Abbreviations \\ CRC: Colorectal cancer; EGFR: Epidermal Growth Factor Receptor; FFPE: Formalin-fixed-parafin-embedded; HE: Haematoxylin and eosin; MSI: Microsatellite instability; smMIP: single-molecule molecular inversion probe}

\section{Acknowledgements}

Not applicable.

\section{Authors' contributions}

Design of the study: CP, IN. Selecting cases: NK, JM, SR, MT, LM. DNA isolation and smMIPs analysis: NK, CvdW, SvV, LM. Analysing data: NK, CvdW, SVV, FS. Figures: CvdW. Manuscript preparation: NK, FS, IN. Feedback manuscript: CvdW, SVV, JM, SR, MT, LM, CP. All authors read and approved the final manuscript.

\section{Funding}

This study was supported by a personal grant from the Dutch Cancer Society (N.K., KUN 2011-5251) and Amgen (20139137).

Availability of data and materials

Iris Nagtegaal is the guarantor of this work and, as such, had full access to all of the data in the study and takes responsibility for the integrity of the data and the accuracy of the data analysis. The datasets used and analyzed 
during the current study are available from the corresponding author on reasonable request.

\section{Ethics approval and consent to participate}

The Institutional Review Board of the Radboud university medical center, Nijmegen, ruled that the current study does not require informed consent according to Dutch law, but based on national guidelines for the use of archival material, the Institutional Review Board agrees with the study proposal (CMO 2013/048, date 23/4/2013).

\section{Consent for publication}

Not applicable

\section{Competing interests}

The authors declare that they have no competing interests.

\section{Author details}

'Department of Pathology, Radboud Institute of Molecular Life Sciences, Radboud University Medical Center, PO Box 9101, Nijmegen 6500 HB, The Netherlands. '2Department of Pathology, Rijnstate Hospital, Arnhem, The Netherlands. ${ }^{3}$ Laboratory of Pathology East Netherlands, Hengelo, The Netherlands. ${ }^{4}$ Department of Medical Oncology, Academic Medical Center, University of Amsterdam, Amsterdam, The Netherlands. ${ }^{5}$ Department of Medical Oncology, Hospital Medisch Spectrum Twente, The Netherlands Oncology, Enschede, Amsterdam, The Netherlands.

Received: 17 May 2019 Accepted: 5 August 2019

Published online: 16 September 2019

\section{References}

1. Karapetis CS, Khambata-Ford S, Jonker DJ, et al. K-ras mutations and benefit from cetuximab in advanced colorectal cancer. N Engl J Med. 2008:359:1757-65.

2. Douillard JY, Oliner KS, Siena S, et al. Panitumumab-folfox4 treatment and ras mutations in colorectal cancer. N Engl J Med. 2013;369:1023-34.

3. Pietrantonio F, Petrelli F, Coinu A, et al. Predictive role of braf mutations in patients with advanced colorectal cancer receiving cetuximab and panitumumab: a meta-analysis. Eur J Cancer. 2015;51:587-94.

4. Knijn N, Mekenkamp LJ, Klomp M, et al. Kras mutation analysis: a comparison between primary tumours and matched liver metastases in 305 colorectal cancer patients. Br J Cancer. 2011;104:1020-6.

5. Hiatt JB, Pritchard CC, Salipante SJ, O'Roak BJ, Shendure J. Single molecule molecular inversion probes for targeted, high-accuracy detection of lowfrequency variation. Genome Res. 2013;23:843-54.

6. Eijkelenboom A, Kamping EJ, Kastner-van Raaij AW, et al. Reliable nextgeneration sequencing of formalin-fixed, paraffin-embedded tissue using single molecule tags. J Mol Diagn. 2016;18:851-63.

7. Suraweera N, Duval A, Reperant M, et al. Evaluation of tumor microsatellite instability using five quasimonomorphic mononucleotide repeats and pentaplex pcr. Gastroenterology. 2002;123:1804-11.

8. Fujiyoshi K, Yamamoto G, Takahashi A, et al. High concordance rate of kras/ braf mutations and msi-h between primary colorectal cancer and corresponding metastases. Oncol Rep. 2017;37:785-92.

9. Mao C, Wu XY, Yang ZY, et al. Concordant analysis of kras, braf, pik3ca mutations, and pten expression between primary colorectal cancer and matched metastases. Sci Rep. 2015;5:8065.

10. Cejas P, Lopez-Gomez M, Aguayo C, et al. Analysis of the concordance in the egfr pathway status between primary tumors and related metastases of colorectal cancer patients: implications for cancer therapy. Curr Cancer Drug Targets. 2012;12:124-31.

11. Mostert B, Jiang Y, Sieuwerts AM, et al. Kras and braf mutation status in circulating colorectal tumor cells and their correlation with primary and metastatic tumor tissue. Int J Cancer. 2013;133:130-41.

12. Kim MJ, Lee HS, Kim JH, et al. Different metastatic pattern according to the kras mutational status and site-specific discordance of kras status in patients with colorectal cancer. BMC Cancer. 2012;12:347.

13. Heerink WJ, de Bock GH, de Jonge GJ, Groen HJ, Vliegenthart R, Oudkerk M. Complication rates of ct-quided transthoracic lung biopsy: meta-analysis. Eur Radiol. 2017;27:138-48.

14. Diaz LA Jr, Williams RT, Wu J, et al. The molecular evolution of acquired resistance to targeted egfr blockade in colorectal cancers. Nature. 2012;486:537-40.
15. Misale $S$, Yaeger R, Hobor $S$, et al. Emergence of kras mutations and acquired resistance to anti-egfr therapy in colorectal cancer. Nature. 2012;486:532-6.

16. Bettegowda C, Sausen M, Leary RJ, et al. Detection of circulating tumor DNA in early- and late-stage human malignancies. Sci Transl Med. 2014;6:224ra224.

17. Cejas P, Lopez-Gomez M, Aguayo C, et al. Kras mutations in primary colorectal cancer tumors and related metastases: a potential role in prediction of lung metastasis. PLoS One. 2009;4:e8199.

18. Pereira AA, Rego JF, Morris V, et al. Association between kras mutation and lung metastasis in advanced colorectal cancer. Br J Cancer. 2015;112:424-8.

19. Lipsyc M, Yaeger R. Impact of somatic mutations on patterns of metastasis in colorectal cancer. J Gastrointest Oncol. 2015:6:645-9.

20. Russo AL, Borger DR, Szymonifka J, et al. Mutational analysis and clinical correlation of metastatic colorectal cancer. Cancer. 2014:120:1482-90.

21. Tran B, Kopetz S, Tie J, et al. Impact of braf mutation and microsatellite instability on the pattern of metastatic spread and prognosis in metastatic colorectal cancer. Cancer. 2011;117:4623-32.

22. Venderbosch S, Nagtegaal ID, Maughan TS, et al. Mismatch repair status and braf mutation status in metastatic colorectal cancer patients: a pooled analysis of the Cairo, cairo2, coin, and focus studies. Clin Cancer Res. 2014;20:5322-30.

23. Koopman M, Kortman GA, Mekenkamp L, et al. Deficient mismatch repair system in patients with sporadic advanced colorectal cancer. Br J Cancer. 2009;100:266-73.

24. Wong SQ, Li J, Tan AY, et al. Sequence artefacts in a prospective series of formalin-fixed tumours tested for mutations in hotspot regions by massively parallel sequencing. BMC Med Genet. 2014;7:23.

25. Ludyga N, Grunwald B, Azimzadeh O, et al. Nucleic acids from long-term preserved ffpe tissues are suitable for downstream analyses. Virchows Arch. 2012:460:131-40.

\section{Publisher's Note}

Springer Nature remains neutral with regard to jurisdictional claims in published maps and institutional affiliations.
Ready to submit your research? Choose BMC and benefit from:

- fast, convenient online submission

- thorough peer review by experienced researchers in your field

- rapid publication on acceptance

- support for research data, including large and complex data types

- gold Open Access which fosters wider collaboration and increased citations

- maximum visibility for your research: over $100 \mathrm{M}$ website views per year

At $\mathrm{BMC}$, research is always in progress.

Learn more biomedcentral.com/submissions 